\title{
Actitud de universitarios hacia las personas con discapacidad
}

\section{Alexis Araya-Cortés}

Universidad de La Serena, Chile alexisarayacortes@gmail.com

\section{Mauricio González-Arias}

Universidad de La Serena, Chile mgonzale@userena.cl

\section{Carola Cerpa-Reyes}

Universidad de La Serena, Chile cacerpa@gmail.com

\section{Resumen}

El objetivo de este estudio fue describir y comparar la actitud hacia las personas con discapacidad de estudiantes de pedagogía, con estudiantes de otras profesiones y en distintos niveles de formación. Se utilizó un diseño descriptivo y correlacional en el que participaron 260 estudiantes de ambos sexos de una universidad privada de la cuidad de La Serena, Chile. Como instrumento se aplicó la Escala de Actitudes hacia las Personas con Discapacidad de Verdugo, Jenaro y Arias. Los resultados mostraron que la totalidad del alumnado presenta actitudes positivas. En relación con el tipo de carrera y nivel de estudio no se encontraron diferencias estadísticamente significativas. Se puede inferir que los estudiantes presentan una predisposición favorable a comportarse de manera efectiva frente a personas con discapacidad, valorando positivamente sus capacidades y reconociendo sus derechos fundamentales.

\section{Palabras clave}

Actitud del estudiante, educación, estudiante universitario, incapacitado. (Fuente: Tesauro de la Unesco).

Recepción: 2014-03-11 / Envío a pares: 2014-06-20 / Aceptación por pares: 2014-07-01 / Aprobación: 2014-07-15 DOI: $10.5294 /$ edu.2014.17.2.5

Para citar este artículo / To reference this article / Para citar este artigo

Araya-Cortés, A., González-Arias, M. y Cerpa-Reyes, C. (2014). Actitud de universitarios hacia las personas con discapacidad. Educ. Educ. 17 (2), 289-305. Doi. 10.5294/edu.2014.17.2.5 


\title{
Attitude of University Students Towards the Disabled
}

\begin{abstract}
The objective of this study is to describe and compare the attitudes towards disabled persons found among students majoring in education and those in other professions, and at different levels of training. A descriptive correlational design was used on a sample of 260 male and female students from a private university in the city of La Serena, Chile. The Attitudes toward Disabled Persons Scale was applied, as developed by Verdugo, Januarius and Arias (1995). The results show all the students demonstrate positive attitudes. As for college major or discipline and level of study, no statistically significant differences were found. Therefore, it is possible to infer that students are favorably predisposed to behaving effectively with people who have disabilities, by assessing their capabilities positively and acknowledging their fundamental rights.
\end{abstract}

\section{Key words}

Student's attitude, education, college student, disabled. (Source: Unesco Thesaurus). 


\section{Atitude de universitários quanto a pessoas com deficiência}

Resumo

O objetivo deste estudo foi descrever e comparar a atitude quanto às pessoas com deficiência de estudantes de pedagogia, com estudantes de outras profissões e em diferentes niveis de formação. Utilizou-se um desenho descritivo e correlacional do qual participaram 260 estudantes de ambos os sexos de uma universidade particular da cidade de La Serena, Chile. Como instrumento, aplicou-se a Escala de Atitudes quanto às Pessoas com Deficiência de Verdugo, Jenaro e Arias (1995). Os resultados mostraram que a totalidade do alunado apresenta atitudes positivas. A respeito do tipo de curso acadêmico e nível de estudo, não se constataram diferenças estatisticamente significativas. Pode-se inferir que os estudantes apresentam uma predisposição favorável a se comportarem de maneira efetiva ante pessoas com necessidades especiais, valorizam positivamente suas capacidades e reconhecem seus direitos fundamentais.

\section{Palavras-chave}

Atitude do estudante, educação, estudante universitário, pessoa com deficiência. (Fonte: Tesauro da Unesco). 


\section{Introducción}

La actitud hacia las personas con discapacidad es un tema que ha cobrado relevancia en los últimos años, a partir de un mayor grado de conciencia social por mejorar la calidad de vida de estos individuos. Las actitudes y expectativas negativas hacia las personas con discapacidad siguen siendo en la actualidad motivo de análisis, puesto que constituyen una de las principales barreras para su inclusión e integración (Polo, Fernández y Díaz, 2011).

Dentro de los ámbitos relevantes para mejorar la calidad de vida de personas con discapacidad se encuentra el contexto educacional. En Chile se han desarrollado políticas específicas orientadas en esta línea, aunque aún queda mucho camino por recorrer para asegurar que los alumnos con discapacidad puedan recibir una educación de calidad y en condiciones de equidad (Lissi et al., 2009).

Asimismo, en el año 2010 entró en vigencia la Ley 20.422, la cual establece normas sobre igualdad de oportunidades e inclusión social de las personas con discapacidad. Esta ley hace referencia de manera explícita a la educación e inclusión, y señala que el Estado debe garantizar a las personas con discapacidad el acceso a los establecimientos públicos y privados del sistema de educación regular o a los establecimientos de educación especial, según corresponda.

Debido a la necesidad de mejorar el sistema de educación especial, en el año 2010 se promulga el Decreto Supremo 170, el cual fija normas para determinar los alumnos con necesidades educativas especiales que serán beneficiarios de la subvención para educación especial, estableciendo los requisitos, las evaluaciones y los perfiles de los profesionales competentes que deberán llevar a cabo este proceso.

Todos estos cambios de normativas hacia la discapacidad en ámbitos educativos y sociales han generado una reorganización del sistema interno de las instituciones educativas, modificando la cultura, las políticas y las prácticas pedagógicas. Este conjunto de medidas favorece el proceso de integra- ción de este colectivo de personas, pero no asegura el éxito en el proceso, ya que en este intervienen una serie de variables, entre las cuales se encuentran las actitudes hacia las personas con discapacidad (Mella y González, 2007). En relación con lo anterior, la investigación reciente señala que las actitudes son un aspecto fundamental para el éxito de las personas con discapacidad en sus procesos de integración y en aquellos de enseñanza-aprendizaje (Bueno, 2010; Jarvis y French, 1990; Verdugo, Arias y Jenaro, 1994).

En la misma línea, dentro de los factores que han sido considerados altamente relevantes para el logro de la integración escolar se encuentran las actitudes o la disposición del docente hacia esta alternativa de educación y hacia los estudiantes con necesidades educativas especiales asociadas a discapacidad (Álvarez, Campo, Castro y Martino, 2005). De forma simultánea, la predisposición de los profesores hacia la integración de los alumnos con discapacidad constituye un factor condicionante de los resultados que se obtienen, por ello una actitud positiva es ya un primer paso importante que facilita la educación de estos alumnos en la escuela integradora (Lucchini, 2003). En este sentido, la formación de profesores cobra aún mayor relevancia porque son ellos quienes materializan o hacen posible la integración y futura inclusión en las instituciones educativas y posteriormente en la sociedad.

Dados los nuevos desafíos que tienen que asumir tanto los docentes de las escuelas comunes - que deben responder a una mayor diversidad de necesidades del alumnado-como los de educación especial - cuyo escenario de trabajo y rol cambian considerablemente-, su formación inicial y continua es de máxima importancia para avanzar en el desarrollo de prácticas y culturas escolares más inclusivas (Duk y Murillo, 2010).

Según Duk y Murillo (2010), formar profesores con competencias para trabajar en contextos y con estudiantes cada vez más complejos y heterogéneos es el gran reto que enfrentan las instituciones formadoras del profesorado en los países latinoa- 
mericanos. Repensar los perfiles profesionales y los modelos formativos de cara a las transformaciones que exige una pedagogía basada en los principios de inclusión y atención a la diversidad, en el contexto de los aprendizajes que demanda el siglo XXI, es una necesidad urgente si se aspira alcanzar la meta de educación de calidad para todos, sin exclusiones ni discriminaciones de ningún tipo. No obstante, al considerar la importancia de las actitudes de los docentes hacia la discapacidad existen pocos estudios que describan cómo son las de los estudiantes de pedagogía, y si éstas son similares o diferentes a los estudiantes de otras carreras universitarias. Además, no hay estudios que muestren si al avanzar desde los primeros a los últimos años en educación superior se produce algún cambio en estas actitudes.

En este mismo sentido, la investigación cobra relevancia debido a que las actitudes hacia la discapacidad pueden condicionar, en gran medida, el grado de inclusión o exclusión de las personas con discapacidades dentro de la sociedad (Alemany y Villuendas, 2004). A partir de las actitudes hacia estas personas se puede trabajar a fin de cambiarlas cuando son negativas, o reforzar las positivas para lograr mayor integración escolar y social.

Conocer las actitudes de los estudiantes de pedagogía, en el contexto de una universidad regional en Chile, tiene además la relevancia de proveer información específica para posteriores estudios comparativos a nivel nacional e internacional. Asimismo, esta información proporcionará una base para la reflexión en relación con los perfiles de egreso de las carreras de pedagogía y su coherencia con las políticas públicas y demandas sociales sobre el tema.

\section{La actitud y el comportamiento}

La actitud ha sido definida bajo una gran gama de conceptos que son utilizados en el lenguaje cotidiano, a veces de forma errónea, lo que puede dar lugar a confusiones. Por esto es necesario aclarar el término. Sin embargo, resulta difícil debido a que son numerosas las definiciones que se han dado.
Desde la teoría de la acción favorable (Ajzen y Fishlein, 1980) se entiende por actitud: una evaluación general o un sentimiento global, favorable o no, hacia un comportamiento o cuestión. Las actitudes, en cuanto son evaluaciones afectivas, muestran significado y juicio, por lo que están abiertas al cambio a través de la reflexión y además son educables (Escámez, García, Pérez y Llopis, 2007).

Por otra parte, existen dos principales teorías que explican la relación entre actitud y comportamiento propuesta por Ajzen y Fishbein (1980). La primera de ellas se denomina teoría de la acción razonada, y sus aspectos fundamentales son, en primer lugar, que las intenciones determinan el comportamiento de manera causal y, en segundo lugar, que las intenciones son provocadas por la suma de las influencias de las actitudes hacia el comportamiento y de las normas subjetivas (Novo, Muñoz y Calvo, 2011). La segunda teoría se denomina acción planificada, la cual nace al agregar un nuevo componente a la teoría anterior: el control percibido de la conducta. Este componente guarda relación con el hecho de percibir obstáculos externos o internos que impiden o dificultan que se lleve a cabo la conducta (Morales et al., 1999).

\section{Estudios sobre la actitud hacia la discapacidad}

Diferentes estudios han investigado las actitudes hacia personas con discapacidad en diversos colectivos sociales: profesores, profesionales de la salud o compañeros de clase (Alemany y Villuendas, 2004; Quintero, Salazar, Padrón y Salazar, 2005; Moreno, Aguilera, Rodríguez y Saldaña, 2006). Una serie de investigaciones han comprobado que existen diferencias en las actitudes hacia personas con discapacidad en función de la condición de poseer o no contacto con ellas (López, 2006; Polo et al., 2011).

En este sentido, en un estudio llevado a cabo en Chile por Gómez e Infante (2004) se examinaron las actitudes mostradas por estudiantes universi- 
tarios de diversas carreras. Los resultados indicaron que eran más positivas en aquellos del último curso. Además, estos autores señalan que la mejor actitud observada es la de estudiantes de Pedagogía. En este mismo sentido, Alonso, Navarro y Lidón (2004) llevaron a cabo un estudio sobre la actitud hacia la diversidad con objeto de analizar si existen diferencias en estas actitudes en función del área de conocimiento de los estudiantes universitarios, seleccionando cinco áreas diversas (Educación, Humanidades, Ciencias Experimentales, Ciencias Sociales e Ingeniería). Sus resultados revelan diferencias en función de esta variable, de manera que las actitudes hacia la discapacidad son más positivas en los estudiantes de Humanidades y Educación que en el resto de áreas de conocimiento.

También, con fines comparativos, Gughwan y Chow (2001) examinaron las actitudes hacia la diversidad en varios grupos de estudiantes de diferentes etapas. Los resultados indicaron que los estudiantes de posgrado presentaban actitudes más favorables hacia la integración de personas con discapacidad que los de pregrado.

Las recientes investigaciones señalan que estudiantes universitarios de sexo femenino tenderían a puntuar más favorablemente en escalas de actitudes hacia las personas con discapacidad que los hombres (Findler, Vilchinsky y Werner, 2007; Upton y Harper, 2002). Pero a la vez, otros estudios (Moreno et al., 2006) reportan que no existirían diferencias significativas en las actitudes en función del género.

Una de las pocas variables sociodemográficas en las que habría resultados más concluyentes es el contacto o la experiencia con la discapacidad, en la que a un mayor contacto existirían actitudes más favorables hacia individuos con discapacidad (Hunty Hunt, 2000, en Barr y Bracchita, 2008). Se ha planteado que sería el contacto con personas con discapacidad lo que permitiría reducir estereotipos (Yuker, 1994, en Barr y Bracchita, 2008) y, en esa línea, influir en actitudes más favorables.
Lissi et al. (2009) señalan que a pesar de las investigaciones anteriores, Rao (2004) enfatiza que los resultados respecto a la importancia del contacto no son concluyentes. A partir de eso pareciera relevante hacer ciertas distinciones en la forma en que se ha medido esta variable. Respecto a los estudiantes, un estudio realizado por Upton y Harper (2002), en donde los alumnos debían escoger aquellos tipos de discapacidad en los que consideraban más justo recibir algún tipo de adecuación, los tipos de discapacidad mencionados más frecuentemente fueron la visual, la parálisis cerebral, el daño cerebral y la discapacidad auditiva.

Más recientemente, Suriá (2011) se centró en la actitud de los estudiantes de diferentes etapas educativas hacia la discapacidad analizando cómo son las actitudes de los alumnos hacia sus compañeros con discapacidad en función de su etapa formativa, así como del curso concreto. El trabajo de Suriá también pretendía comprobar la influencia de la interacción con estas personas sobre la formación de la actitud hacia la discapacidad. Los resultados de su estudio indican que los estudiantes universitarios muestran hacia sus compañeros con discapacidad una actitud más positiva que los alumnos de educación secundaria. Al mismo tiempo, Suriá destaca la existencia de un mayor grado de sensibilización hacia la discapacidad por parte de aquellos estudiantes que interaccionan con compañeros que la tienen.

En consideración a los antecedentes señalados, el objetivo de este estudio fue describir y comparar la actitud hacia las personas con discapacidad de estudiantes de diferentes pedagogías con estudiantes de otras profesiones y en distintos niveles de formación. Específicamente se formularon dos objetivos: 1) describir las actitudes que tienen los estudiantes universitarios hacia la discapacidad, 2) comparar la actitud hacia la discapacidad entre estudiantes de diferentes carreras, niveles y contacto previo con la discapacidad. 


\section{Materiales y método}

La presente investigación tiene un diseño descriptivo y correlacional, con un enfoque cuantitativo para el análisis de los principales resultados.

\section{Participantes}

La muestra del estudio estuvo integrada por 260 estudiantes de una universidad privada de la ciudad de La Serena, los cuales cursaban distintas carreras universitarias en dos niveles de formación $\left(2^{\circ}\right.$ y $4^{\circ}$ año). Los estudiantes se dividieron en dos grupos, el primero lo conformaron los alumnos de las carreras de pedagogía (Educación Diferencial, Educación Básica y Educación Física) y el segundo grupo estuvo conformado por estudiantes de otras profesiones (Trabajo Social, Psicología, Enfermería y Educación Parvularia). La muestra estaba compuesta en su mayoría por estudiantes de las carreras de Educación Diferencial (17,7\%) y Psicología (17,3\%), siendo la menor proporción de alumnos en la carrera de Educación Parvularia (9,6\%). La mayoría de los participantes fueron mujeres, y por la naturaleza de las carreras en algunos casos solo había mujeres. Una visión más detallada de los participantes se puede apreciar en la tabla 1.

Respecto a la muestra, en la tabla 2 se describen las frecuencias de participantes según la razón del contacto previo y la asiduidad del contacto previo con personas con discapacidad. La razón más frecuente es la familiar y la frecuencia es principalmente esporádica pero también hay cantidades importantes de participantes con contacto frecuente y permanente.

\section{Instrumentos}

El instrumento utilizado para realizar el estudio fue la Escala de Actitudes hacia las Personas con Discapacidad, Forma G (EAPD) de Verdugo, Arias y Jenaro (1995). Se trata de una escala multidimensional para la evaluación de actitudes ante personas con cualquier tipo de discapacidad.

Para responder al instrumento el participante debe opinar si está de acuerdo o no con los enunciados que se presentan, formulados positiva y negativamente, siendo los significados de las opiniones los siguientes: Estoy muy de acuerdo (MA), Estoy bastante de acuerdo (BA), Estoy parcialmente de acuerdo (PA), Estoy parcialmente en desacuerdo (PD), Estoy en bastante desacuerdo (BD) y Estoy muy en desacuerdo (MD).

La EAPD está formada por 37 ítems, los cuales integran cinco subescalas: 1) Valoración de capacidades y limitaciones (ítems 1, 2, 4, 7, 8, 16, 21, 29 y 36); 2)

Tabla 1. Distribución de los participantes según variables de contexto

\begin{tabular}{|c|c|c|c|c|c|c|c|c|c|c|c|}
\hline \multirow{2}{*}{ Carrera } & \multicolumn{3}{|c|}{ Sexo } & \multicolumn{2}{c|}{$\begin{array}{c}\text { Categoría edad } \\
\text { en años }\end{array}$} & \multicolumn{3}{c|}{ Nivel } & \multicolumn{2}{c|}{$\begin{array}{c}\text { Ha tenido } \\
\text { asignatura sobre } \\
\text { discapacidad }\end{array}$} & \multicolumn{2}{c|}{$\begin{array}{c}\text { Ha tenido contacto } \\
\text { con discapacidad }\end{array}$} \\
Total \\
\hline & Mujer & Hombre & 18 a 20 & 21 a 40 & Segundo & Cuarto & Si & No & Si & No & \\
\hline Educación Diferencial & 46 & 0 & 15 & 31 & 21 & 25 & 15 & 31 & 28 & 18 & 46 \\
\hline Educación Básica & 28 & 2 & 3 & 27 & 10 & 20 & 19 & 11 & 13 & 17 & 30 \\
\hline $\begin{array}{c}\text { Pedagogía en } \\
\text { Educación Física }\end{array}$ & 16 & 26 & 21 & 21 & 23 & 19 & 6 & 36 & 23 & 19 & 42 \\
\hline Trabajo Social & 28 & 2 & 12 & 18 & 16 & 14 & 0 & 30 & 27 & 3 & 30 \\
\hline Psicología & 34 & 11 & 24 & 21 & 28 & 17 & 7 & 38 & 23 & 22 & 45 \\
\hline Enfermería & 38 & 4 & 18 & 24 & 23 & 19 & 0 & 42 & 26 & 16 & 42 \\
\hline Educación Parvularia & 25 & 0 & 5 & 20 & 10 & 15 & 15 & 10 & 13 & 12 & 25 \\
\hline Total & 215 & 45 & 98 & 154 & 131 & 129 & 62 & 198 & 153 & 107 & 260 \\
\hline
\end{tabular}


Tabla 2. Cantidad de participantes por carrera según el motivo y la frecuencia de contacto previo con discapacitados

\begin{tabular}{|l|c|c|c|c|c|c|c|c|}
\hline \multirow{2}{*}{ Carrera } & \multicolumn{4}{c|}{ Razón o motivo del contacto previo con } & \multicolumn{3}{c|}{$\begin{array}{c}\text { Frecuencia de contacto con } \\
\text { discapacidad }\end{array}$} \\
\cline { 2 - 12 } & Familiar & Laboral & Asistencial & Amistad & Otras & Permanente & Frecuente & Esporádico \\
\hline Educación Diferencial & 13 & 3 & 1 & 3 & 8 & 6 & 7 & 9 \\
\hline Educación Básica & 6 & 0 & 0 & 5 & 1 & 5 & 6 & 4 \\
\hline Pedagogía en Educación Física & 13 & 4 & 0 & 4 & 1 & 6 & 7 & 7 \\
\hline Trabajo Social & 12 & 3 & 0 & 8 & 3 & 6 & 12 & 10 \\
\hline Psicología & 13 & 3 & 1 & 2 & 3 & 5 & 8 & 11 \\
\hline Enfermería & 15 & 1 & 3 & 4 & 2 & 6 & 7 & 12 \\
\hline Educación Parvularia & 8 & 1 & 0 & 3 & 1 & 3 & 6 & 7 \\
\hline Total & 80 & 15 & 5 & 29 & 19 & 37 & 53 & 60 \\
\hline
\end{tabular}

Reconocimiento/negación de los derechos (ítems 6, 9, 12, 13, 14, 15, 22, 23, 27, 35 y 37); 3) Implicación personal (items 3, 5, 10, 11, 25, 26, 31); 4) Calificación genérica (ítems 18, 24, 28 y 34); 5) Asunción de roles (ítems 19, 30 y 33). Los ítems que expresan valoración negativa $(1,2,4,5,6,7,8,10,15,17,18,23,24,25,26,28,29,31,34$, $35,37)$ se codificaron de manera inversa en cuanto a los valores que les asigna la escala. Una puntuación más próxima a 6 para cualquier ítem de la escala refleja una actitud positiva hacia las personas con discapacidad.

Además de los ítems que componen el instrumento se incluyeron preguntas para recoger datos sociodemográficos relacionados con sexo, edad, carrera y nivel de la carrera. Se les pregunta si han cursado alguna asignatura relacionada con la discapacidad y si han tenido contacto con personas con discapacidad. En caso afirmativo del contacto se pregunta acerca de la razón (familiar, laboral, asistencia, amistad u otras razones) y su frecuencia (casi permanente, frecuente o esporádica).

Como el instrumento de evaluación fue construido en un contexto diferente (España) al de su aplicación (La Serena, Chile), para constatar su confiabilidad se calculó el coeficiente de consistencia interna alfa de Cronbach para el total de la prueba que fue de 0,77 , el cual se interpreta como una con- fiabilidad aceptable para este tipo de estudios (Cohen y Mark, 2001).

\section{Procedimiento}

En primera instancia se solicitó, a través de una carta formal, la autorización para realizar dicha investigación a las autoridades correspondientes de la institución de educación superior. La participación de los estudiantes universitarios en esta investigación se decidió sobre la base del objetivo general del estudio, en donde se dio énfasis a las carreras relacionadas con educación y que contaran con niveles comprendidos entre $2^{\circ}$ y $4^{\circ}$ año. En segundo lugar, se seleccionaron otras carreras que cumplían con el criterio del nivel cursado. Una vez elegidos los grupos se planificó con cada jefe de Carrera la aplicación del instrumento, informando vía correo electrónico a todos los docentes de los cursos seleccionados. La escala fue aplicada de manera colectiva por el investigador y un colaborador, manteniendo un protocolo de aplicación que consistía en explicar a los participantes el objetivo de la investigación, leer y explicar las instrucciones de la escala, y solicitar el consentimiento a través de la firma en un apartado del instrumento. Al finalizar la administración los estudiantes tuvieron la posibilidad de realizar preguntas y comentarios, con el propósito de considerar sus aportes al estudio. 


\section{Resultados}

\section{Actitud hacia las personas con discapacidad}

Como se observa en la tabla 3, todas las subescalas tuvieron un promedio de actitud superior al promedio teórico esperado $(M$. [teórico] $=3,5)$ en una escala de 6 puntos. La puntuación más alta fue para la subescala de Implicación personal $(M=5,8$; $D S=0,3)$, seguida de Reconocimiento de derechos ( $M$ $=5,4 ; D S=0,4)$ y Asunción de roles $(M=5,2 ; D S=0,7)$. Dentro de las puntuaciones bajas se encuentra la subescala de Valoración de capacidades y limitaciones $(M=4,9 ; D S=0,6)$. Sin embargo, la puntuación más baja fue para Calificación genérica $(M=4,5$; $D S=0,9)$. En relación con la actitud total hacia las personas con discapacidad, los puntajes promedios indican que los estudiantes presentan una actitud positiva en general $(M=5,2 ; D S=0,4)$.

\section{Actitud hacia las personas con discapacidad en carreras de pedagogía, con respecto a otras carreras}

Después de realizar el análisis descriptivo de los diferentes factores que componen la escala de actitud hacia las personas con discapacidad se realizó un análisis de comparación de medias para muestras independientes, entre estudiantes de carreras de pedagogía (Educación Diferencial, Educación Básica y Pedagogía en Educación Física) y la media obtenida por estudiantes de otras carreras (Trabajo Social, Educación Parvularia, Enfermería y Psicología). Para ello se utilizó la prueba $t$ de Studens, en donde no se encontraron diferencias estadísticamente significativas en ninguna de las subescalas en relación con el tipo de carreras. En la tabla 4 se observa que para la subescala Valoración de la capacidad y limitaciones se encontró que $t(233)=-0,5$; $p>05$. En Reconocimiento de derechos $t(233)=-0,1$; $p>05$. Implicación personal $t$ (233) = - 0,0; $p>05$. Calificación genérica $t$ (233) = -0,3; $p>05$. Asunción de roles $t(233)=-0,3 ; p>05$. Al analizar las medias globales de la escala en relación con los tipos de carreras no se encontraron diferencias estadísticamente significativas, $t(233)=-0,2 ; p>05$. La misma media presentó para Carreras de pedagogía $M=5,1 ; D S=$ 0,3 ; y para otras carreras $M=5,1 ; D S=0,4$.

\section{Actitud hacia la discapacidad en estudiantes de diferentes carreras}

En la tabla 5 se presentan los resultados del análisis estadístico Anova para estudiar los efectos que la variable tipo de carrera tiene sobre las diferentes subescalas que componen la escala de actitudes hacia las personas con discapacidad. Al examinar la media de la escala total no se encuentran diferencias estadísticamente significativas ( $F$ $=0,745, p=0,613)$. El resultado para las subescalas EADP es similar a la escala total. No obstante, las subescalas que presentaron diferencias estadísticamente significativas fueron Valoración de capacidades y limitaciones ( $F=2,681, p=0,015)$ e Implicación personal $(F=2,352, p=0,31)$.

Para profundizar en el análisis de las subescalas que resultan con diferencias significativas y realizar comparaciones múltiples adicionales a la prueba Anova se realizó un Post hoc utilizando la prueba Tukey (tabla 6). De acuerdo con los resultados existen diferencias significativas en la subescala de valoración de capacidades y limitaciones entre las carreras de Educación Diferencial $(M=5,1)$ y Educación Física $(M=4,8),\left(F=0,3716\left(^{*}\right), p=0,029\right)$. También encontramos estas diferencias en la subescala de Implicación personal, entre las carreras de Educación Diferencial $(M=5,9)$ y Educación Parvularia $(M=$ $5,6),\left(F=0,2655\left(^{*}\right), p=0,017\right)$, y entre la carrera de Enfermería $(M=5,9)$ y Educación Parvularia $(M=5,6)$, $\left(F=0,2710\left({ }^{*}\right), p=0,015\right)$. Para las demás subescalas y carreras no se encontraron diferencias estadísticamente significativas. Sin embargo, todas las carreras en la escala total presentan alguna diferencia entre medias, siendo la más relevante la diferencia entre las carreras de Educación Física ( $M=5,0 ; p=0,80$ ) y Educación Diferencial $(M=5,19, p=0,80)$, las cuales presentan una actitud más favorable hacia las personas con discapacidad que el resto de las carreras. 
ISSN 0123-1294 | Educ.Educ. Vol. 17. No. 2 | Mayo-Agosto de 2014 | pp. 289-305.

Universidad de La Sabana | Facultad de Educación

Tabla 3. Estadísticos descriptivos de la escala Actitud hacia las Personas con Discapacidad $(\mathbf{n}=\mathbf{2 6 0})$

\begin{tabular}{|c|c|c|c|c|}
\hline Subescalas EAPD & Mínimo & Máximo & Media & Desv. típ. \\
\hline Valoración de capacidades y limitaciones & 3,3 & 6,0 & 4,9 & 0,6 \\
\hline Reconocimiento/Negación de los derechos & 3,8 & 6,0 & 5,4 & 0,4 \\
\hline Implicación personal & 4,4 & 6,0 & 5,8 & 0,3 \\
\hline Calificación genérica & 2,0 & 6,0 & 4,5 & 0,9 \\
\hline Asunción de roles & 1,0 & 6,0 & 5,2 & 0,7 \\
\hline Actitud hacia la discapacidad total & 4,1 & 6,0 & 5,2 & 0,4 \\
\hline
\end{tabular}

Tabla 4. Comparación de medias y desviación típica según subescalas, carreras de pedagogía y otras carreras

\begin{tabular}{|l|c|c|c|c|c|c|}
\hline \multicolumn{1}{|c|}{ Subescalas EAPD } & Tipo de carrera & Media & Desviación típica. & $\mathbf{t}$ & $\mathrm{gl}$ & Sig. (bilateral) \\
\hline Valoración de la capacidad y limitaciones & Pedagogías & 4,9 & 0,5 & $-0,5$ & 233 & 0,6 \\
\hline & Otras & 5 & 0,6 & & & \\
\hline Reconocimiento de derechos & Pedagogías & 5,4 & 0,4 & $-0,1$ & 233 & 0,947 \\
\hline & Otras & 5,4 & 0,4 & & & \\
\hline Implicación personal & Pedagogías & 5,8 & 0,3 & 0 & 233 & 0,994 \\
\hline & Otras & 5,8 & 0,3 & & & \\
\hline Calificación genérica & Pedagogías & 4,5 & 0,8 & $-0,3$ & 233 & 0,732 \\
\hline & Otras & 4,5 & 0,9 & & & \\
\hline Asunción de roles & Pedagogías & 5 & 0,9 & 0,3 & 233 & 0,729 \\
\hline & Otras & 4,9 & 0,8 & & & 233 \\
\hline Total & Pedagogías & 5,1 & 0,3 & $-0,2$ & 2,861 \\
\hline & Otras & 5,1 & 0,4 & & & \\
\hline
\end{tabular}

Tabla 5. Anova según subescalas y tipos de carrera

\begin{tabular}{|c|c|c|c|c|c|c|}
\hline Subescalas EAPD & & Suma de cuadrados & gl & Media cuadrática & $\mathrm{F}$ & Sig. \\
\hline \multirow{3}{*}{ Valoración de capacidades y limitaciones } & Intergrupos & 4,90 & 6 & 0,817 & 2,681 & 0,015 \\
\hline & Intragrupos & 77,10 & 253 & 0,305 & & \\
\hline & Total & 82,00 & 259 & & & \\
\hline \multirow{3}{*}{ Reconocimiento de derechos } & Intergrupos & 1,54 & 6 & 0,257 & 1,341 & 0,239 \\
\hline & Intragrupos & 48,51 & 253 & 0,192 & & \\
\hline & Total & 50,06 & 259 & & & \\
\hline \multirow{3}{*}{ Implicación personal } & Intergrupos & 1,45 & 6 & 0,241 & 2,352 & 0,031 \\
\hline & Intragrupos & 25,93 & 253 & 0,102 & & \\
\hline & Total & 27,37 & 259 & & & \\
\hline \multirow{3}{*}{ Calificación genérica } & Intergrupos & 1,23 & 6 & 0,205 & 0,272 & 0,949 \\
\hline & Intragrupos & 191,13 & 253 & 0,755 & & \\
\hline & Total & 192,36 & 259 & & & \\
\hline \multirow{3}{*}{ Asunción de roles } & Intergrupos & 2,31 & 6 & 0,385 & 0,575 & 0,750 \\
\hline & Intragrupos & 169,45 & 253 & 0,670 & & \\
\hline & Total & 171,76 & 259 & & & \\
\hline \multirow{3}{*}{ Escala total } & Intergrupos & 0,64 & 6 & 0,106 & 0,745 & 0,613 \\
\hline & Intragrupos & 35,93 & 253 & 0,142 & & \\
\hline & Total & 36,56 & 259 & & & \\
\hline
\end{tabular}




\section{Actitud hacia la discapacidad entre estudiantes de pedagogía de $2^{\circ}$ y $4^{\circ}$ año}

Para comparar la actitud hacia la discapacidad entre estudiantes de pedagogía de $2^{\circ}$ y $4^{\circ}$ año de la carrera se utilizó la prueba t de Student, en donde no se encontraron diferencias estadísticamente significativas en ninguna de las subescalas. En la tabla 7 se observa que para la subescala Valoración de la capacidad y limitaciones se encontró que $t(116)=-0,5 ; p>$ 05; en Reconocimiento de derechos $t(116)=-0,4 ; p>$ 05. Implicación personal $t(116)=-0,9 ; p>05$. Calificación genérica $t(233)=-0,4 ; p>05$. Asunción de roles $t(116)=-1,0 ; p>05$. Al analizar las medias globales de la escala total en relación con el nivel cursado, no se encontraron diferencias estadísticamente significativas, $t(116)=-0,5 ; p>05$. Cabe señalar que la media obtenida en ambos niveles es la misma $(M=5,1)$.

\section{Contacto con personas con discapacidad}

Al analizar las diferencias en función del contacto con personas con discapacidad, como se muestra en la tabla 8, se encontró que los estudiantes universitarios no presentan diferencias estadísticamente

Tabla 6. Comparaciones múltiples HSD de Tukey de subescalas y carreras

\begin{tabular}{|c|c|c|c|c|c|}
\hline Variable dependiente & Carrera* & Carrera & $\begin{array}{c}\text { Diferencia de } \\
\text { medias (I-J) }\end{array}$ & Error típico & Sig. \\
\hline Valoración de capacidades y limitaciones & $\begin{array}{c}\text { Educación } \\
\text { Diferencial }\end{array}$ & $\begin{array}{c}\text { Educación } \\
\text { Física }\end{array}$ & $0,3716\left(^{*}\right)$ & 0,11781 & 0,029 \\
\hline Implicación personal & $\begin{array}{c}\text { Educación } \\
\text { Diferencial }\end{array}$ & $\begin{array}{c}\text { Educación } \\
\text { Parvularia }\end{array}$ & $\left.0,2655^{*}\right)$ & 0,07954 & 0,017 \\
\hline Implicación personal & Enfermería & $\begin{array}{c}\text { Educación } \\
\text { Parvularia }\end{array}$ & $0,2720\left(^{*}\right)$ & 0,08087 & 0,015 \\
\hline
\end{tabular}

${ }^{*}$ Nota: solo se muestran las carreras que resultaron con diferencias significativas.

Tabla 7. Comparación de Medias y desviación típica según subescalas y niveles de estudio cursado de estudiantes de pedagogía

\begin{tabular}{|c|c|c|c|c|c|c|}
\hline \multicolumn{7}{|c|}{ Estadísticos de grupo } \\
\hline Subescalas EAPD & Nivel & Media & Desviación típ. & $\mathrm{t}$ & gl & Sig. (bilateral) \\
\hline \multirow{2}{*}{ Valoración de capacidades y limitaciones } & 2 nivel & 4,9 & 0,5 & $-0,5$ & 116,0 & 0,625 \\
\hline & 4 nivel & 4,9 & 0,5 & & & \\
\hline \multirow{2}{*}{ Reconocimiento de derechos } & 2 nivel & 5,5 & 0,4 & 0,4 & 116,0 & 0,680 \\
\hline & 4 nivel & 5,4 & 0,4 & & & \\
\hline \multirow{2}{*}{ Implicación personal } & 2 nivel & 5,8 & 0,3 & $-0,9$ & 116,0 & 0,351 \\
\hline & 4 nivel & 5,8 & 0,3 & & & \\
\hline \multirow{2}{*}{ Calificación genérica } & 2 nivel & 4,5 & 0,7 & 0,4 & 116,0 & 0,702 \\
\hline & 4 nivel & 4,4 & 0,9 & & & \\
\hline \multirow{2}{*}{ Asunción de roles } & 2 nivel & 5,0 & 0,7 & 1,0 & 116,0 & 0,336 \\
\hline & 4 nivel & 4,9 & 1,0 & & & \\
\hline \multirow[t]{2}{*}{ Actitud discapacidad } & 2 nivel & 5,1 & 0,3 & 0,5 & 116,0 & 0,629 \\
\hline & 4 nivel & 5,1 & 0,4 & & & \\
\hline
\end{tabular}

Nota: se consideran solo estudiantes de pedagogía $(n=118)$. 
Tabla 8. Comparación de medias y desviación estándar, según contacto con discapacidad

\begin{tabular}{|c|c|c|c|c|c|c|}
\hline \multicolumn{7}{|c|}{ Estadísticos de grupo } \\
\hline Subescalas & Contacto & Media & Desviación típ. & $t$ & gl & Sig. (bilateral) \\
\hline \multirow{2}{*}{ Valoración de capacidades y limitaciones } & Sí & 5,0 & 0,6 & 0,65 & 257,0 & 0,514 \\
\hline & No & 4,9 & 0,6 & & & \\
\hline \multirow{2}{*}{ Reconocimiento de derechos } & Sí & 5,4 & 0,4 & 0,18 & 257,0 & 0,859 \\
\hline & No & 5,4 & 0,5 & & & \\
\hline \multirow{2}{*}{ Implicación personal } & Sí & 5,8 & 0,3 & 2,02 & 257,0 & 0,044 \\
\hline & No & 5,8 & 0,4 & & & \\
\hline \multirow{2}{*}{ Calificación genérica } & Sí & 4,5 & 0,9 & $-0,41$ & 257,0 & 0,681 \\
\hline & No & 4,5 & 0,8 & & & \\
\hline \multirow{2}{*}{ Asunción de roles } & Sí & 5,2 & 0,8 & 0,60 & 257,0 & 0,548 \\
\hline & No & 5,2 & 0,7 & & & \\
\hline \multirow{2}{*}{ Actitud discapacidad total } & Sí & 5,2 & 0,4 & 0,64 & 257,0 & 0,522 \\
\hline & No & 5,2 & 0,4 & & & \\
\hline
\end{tabular}

significativas en la actitud general hacia las personas con discapacidad. No obstante, se encontraron diferencias significativas en la subescala de implicación personal $t$ (257) = 2,0; $p<05$. No se encontraron relaciones significativas al analizar dentro del subgrupo con experiencia previa, en función del motivo ni la frecuencia.

\section{Discusión}

\section{Actitud hacia las personas con discapacidad}

Los puntajes promedios obtenidos en la escala EAPD se pueden considerar buenos 0 adecuados, al menos desde una mirada teórica, lo que quiere decir que la actitud de los estudiantes hacia las personas con discapacidad es positiva en todas las escalas; no obstante, resaltan dos subescalas: Implicación personal y Reconocimiento de derechos. Lo anterior evidenciaría que la muestra estudiada presentaría una predisposición favorable a actuar y aceptar de manera efectiva a las personas con discapacidad en contextos sociales, laborales y personales. Asimismo, reconocen los derechos fundamentales de las personas con discapacidad con respecto a la accesibilidad, participación en actividades cívicas, posibilidades de ingreso a trabajos y diversas actividades de ocio, entre otras.

Las actitudes predominantemente positivas de los estudiantes confirman la tendencia que se ha observado en estudios similares (Polo et al., 2011; Suriá, 2011; Lissi et al., 2009; Gómez e Infante, 2004; Upton y Harper, 2002; Moreno et al., 2006) en los que también se evidencian actitudes favorables de estudiantes universitarios hacia personas con discapacidad.

Es importante señalar que los resultados son muy valiosos por cuanto pueden evidenciar que los diferentes esfuerzos por promover el respeto, el reconocimiento de derechos, la inclusión educativa y la participación en diversos contextos sociales, la igualdad de capacidades y oportunidades de las personas que presentan discapacidad a lo largo de unos pocos años están dando resultados. Sin embargo, para comprobarlo se requieren otros estudios que comparen muestras de diferentes generaciones. 


\section{Actitud hacia las personas con discapacidad según la carrera}

En relación con los resultados obtenidos en la comparación de las actitudes hacia las personas con discapacidad que tienen los estudiantes de las carreras de pedagogía, respecto a estudiantes de otras carreras, podemos señalar que no existen diferencias estadísticamente significativas. Es importante destacar que ambos grupos de estudiantes presentan actitudes favorables en todas las subescalas evaluadas y en el puntaje promedio de la escala total. Estos resultados coinciden con los obtenidos por Moreno et al. (2006).

Sin embargo, encontramos estudios cuyos resultados muestran diferencias significativas en las actitudes hacia la discapacidad de estudiantes de carreras de pedagogía respecto a estudiantes de otras carreras (Gómez e Infante, 2004; Polo et al., 2011). En este trabajo los resultados podrían explicarse debido a que los estudiantes de otras carreras (Trabajo social, Educación Parvularia, Enfermería y Psicología), dentro de sus perfiles y mallas curriculares, incorporan asignaturas de formación general relacionadas con culturas y valores, persona y sentido, entre otras. Por otra parte, es importante destacar que la visión de la universidad declara la formación de personas con un nivel de preparación profesional, en valores y actitudes que les permita desempeñarse con integridad, eficiencia, creatividad y responsabilidad social, contribuyendo al desarrollo de su familia y comunidad, aportando a la cultura y a la generación de nuevos conocimientos.

De manera más específica, de acuerdo con los resultados obtenidos en la comparación de la actitud hacia la discapacidad de distintas carreras, no se observaron diferencias estadísticamente significativas en la escala total y los tipos de carrera. Al realizar un análisis múltiple de las carreras y subescalas se encontraron diferencias estadísticas en la subescala de valoración de capacidades y limitaciones entre las carreras de Educación Diferencial y Educación Física. La carrera de Educación Diferencial muestra una actitud más adecuada o positiva en relación con esta subescala. Esto quiere decir que los estudiantes valoran de forma positiva las capacidades de las personas con discapacidad y poseen altas expectativas en relación con el funcionamiento cognitivo y el desempeño en general, especialmente en el ámbito laboral. Esta diferencia puede ser entendida por los diferentes perfiles de egreso y mallas curriculares de ambas carreras. Asimismo, del total de alumnos de la carrera de Educación Física, solo el $16 \%$ habría cursado alguna asignatura relacionada con discapacidad.

Por otra parte, encontramos diferencias estadísticamente significativas entre las subescalas y la carrera cursada. Así, observamos que los estudiantes de Educación Parvularia mantienen una actitud menos favorable que las carreras de Educación Diferencial y Enfermería en relación con la subescala Implicación personal. Investigaciones realizadas por Polo et al. (2011) y Polo y López-Justicia (2006) han señalado la existencia de diferencias significativas entre las actitudes manifestadas en función de la titulación cursada. Ahora bien, las diferencias encontradas en este trabajo son a nivel de subescalas y no de escala total. Es relevante destacar que en los resultados generales de la escala EADP los estudiantes de Educación Diferencial presentan una actitud más favorable y positiva en general hacia las personas que tienen algún tipo de discapacidad. Este hecho podría explicarse debido a que estos estudiantes poseen competencias personales al elegir esta carrera y una sólida formación hacia el trabajo con y en la diversidad.

\section{Actitud hacia la discapacidad entre estudiantes de pedagogía de $2^{\circ}$ y $4^{\circ}$ año}

Según los resultados obtenidos se puede señalar que no existen diferencias estadísticamente significativas al comparar la actitud de estudiantes que cursan $2^{\circ}$ y $4^{\circ}$ año de carreras de pedagogía (Educación Diferencial, Educación Básica y Educación Física) hacia las personas que presentan disca- 
pacidad. Similares resultados encontramos en estudios anteriores (Moreno et al., 2006), en los cuales no se encontraron diferencias significativas según el curso o nivel de estudiantes de carreras de magisterio y psicopedagogía. Este resultado va contra lo esperado ya que en las carreras de pedagogía se espera que con el avance curricular los futuros profesores desarrollen una mayor sensibilidad hacia la discapacidad, sobre todo porque las políticas de inclusión propician la incorporación de alumnos discapacitados a clases regulares. Hay que profundizar en este aspecto a fin de incorporar de manera más sistemática e intencionada el incremento de una mejor actitud. También se podría comparar entre carreras que lo tienen definido de manera explícita y otras que no lo tengan.

\section{Contacto previo con personas con discapacidad}

Diversas investigaciones (Polo et al., 2011; Moreno et al., 2006) concluyen que la interacción con personas que presentan discapacidad condicionaría una actitud más favorable hacia ellas. Sin embargo, en este trabajo a nivel de escala total no se encontraron diferencias estadísticamente significativas entre los estudiantes que habían tenido algún tipo de contacto y el alumnado que no había interactuado con personas con discapacidad. No obstante, la subescala de Implicación personal presentó diferencias estadísticas. Este resultado podría dar cuenta de las proyecciones favorables de interactuar con personas con discapacidad.

\section{Conclusiones}

En líneas generales, los datos obtenidos nos permiten concluir que la mayoría de los estudiantes universitarios de esta investigación presentan una actitud muy favorable hacia las personas con discapacidad, independientemente del tipo de carrera y nivel de estudio cursado. Las actitudes positivas que se observan en los participantes se relacionan con una concepción más real y contextualizada del término, un adecuado reconocimiento de los derechos fundamentales de estas personas y una valoración de las capacidades dependiendo de la heterogeneidad de la discapacidad. El alumnado muestra una predisposición a actuar y aceptar de manera efectiva a estas personas. Según Gitlow (2001), lo anterior refleja un diagnóstico favorable en cuanto a las barreras intangibles.

Los datos presentados en la investigación son muy interesantes para la reflexión de los estudiantes y especialmente de la comunidad universitaria, puesto que en la actualidad las instituciones de educación están incorporando a sus proyectos institucionales temas como la accesibilidad y el diseño universal de aprendizaje para dar respuesta y brindar apoyo a las diversas necesidades educativas especiales asociadas a la discapacidad. Asimismo, es importante destacar que en este estudio los profesores en formación mostraron actitudes muy favorables, lo cual es fundamental para su futuro quehacer pedagógico.

El contacto o la interacción con personas con discapacidad, en este trabajo, no fue un indicador significativo para determinar las diferencias en la actitud de los estudiantes universitarios hacia este grupo.

Por último, es relevante señalar ciertas limitaciones del presente estudio. En primer lugar, los resultados aluden a un porcentaje limitado de población universitaria, por lo cual no pueden ser generalizados a otros grupos de estudiantes. No obstante, los resultados son muy alentadores para la institución, ya que presentan un diagnóstico favorable en relación con las barreras intangibles. En segundo lugar, y como consecuencia del instrumento utilizado, no se ha podido obtener información sobre la actitud de los estudiantes hacia una discapacidad específica. Por tanto sería importante, en futuras investigaciones, estudiar una muestra más amplia de universitarios, medir la actitud en relación con las diversas discapacidades y, por otra parte, comparar la actitud de profesores en formación con profesores ya titulados y con algunos años de ejercicio profesional. 


\section{Referencias}

Ajzen, I. y Fishbein, M. (1980). Understanding attitudes and predicting social behavior. Englewood Cliff, New Jersey: Prentice Hall.

Alemany, I. y Villuendas, M. D. (2004). Las actitudes del profesorado hacia el alumnado con necesidades educativas especiales. Convergencia, 11 (34),183-215. Recuperado el 17 de septiembre de 2013 de: http://www. redaly c.org/articulo.oa?id $=10503408$

Alonso, J., Navarro, R. y Lidón, V. (2004). Actitudes hacia la diversidad en estudiantes universitarios. Jornades de Foment de la Investigació. Universitat Jaume I. Recuperado el 6 de octubre de 2013 de: http://www.uji.es/ bin/publ/edicions/jfi13/44.pdf

Álvarez, M., Castro, P., Campo, M. y Martino, E. (2005). Actitudes de los maestros ante las necesidades educativas específicas. Revista Psicothema, 17 (04), 601-606. Recuperado 19 de octubre de 2013 de: http://www.psicothema.com/psicothema.asp?id=3152

Barr, J.y Bracchita, K. (2008). Effects of contact with disabilities: positive attitudes and majoring education. The Journal of Psychology, 142 (3), 225-243.

Bueno, A. (2010). Una mirada ilusionada al futuro de los servicios de apoyo a la discapacidad en la Universidad. Buenas prácticas de apoyo a la discapacidad en la universidad. En Bueno, A. Buenas prácticas durante los estudios universitarios en la recepción de información (pp. 10-30). [Tesis doctoral]. Alicante: CEE Limencop. primaria. Universidad de Oviedo.

Cantero, J. León, M. y Barriga, S. (1998). Actitudes: naturaleza, formación y cambio. En León, J. M. et al. (coords.). Psicología Social. Orientaciones teóricas y ejercicios prácticos (pp. 117-132). Madrid: McGraw-Hill.

Cohen, R. J., y Mark, E. S. (2001). Pruebas y evaluación Psicológica. Introducción a las pruebas y a la medición. México: McGraw Hill.

Duk, C. y Murillo J. (2010). Claves de la formación de profesores para escuelas inclusivas. Revista Latinoamericana de Educación Inclusiva, 4 (02), 11-12. Recuperado 8 de noviembre de 2013 de: http://www.rinace.net/rlei/ numeros/vol3-num2/Rev.\%20Ed.\%2olnc.\%20Vol3,2.pdf

Escámez, J., García, R., Pérez, C. y Llopis, A. (2007). El aprendizaje de valores y actitudes. Teoría y práctica (Colección Educación en Valores). Madrid: Octaedro/OEl. Recuperado 11 de noviembre de 2013 de: http://www.rieoei. org/recensiones/GilMadrona451.pdf

Findler, L., Vilchinsky, N. y Werner, S. (2007). The multidimensional attitudes scale toward persons with disabilities (MAS). Rehabilitation Counseling Bulletin, 50, 166-177. Recuperado 10 de octubre de 2013 de: http://rcb. sagepub.com/content/50/3/166.short

Gitlow, L. (2001). Occupational Therapy Faculty Attitudes toward the Inclusion of Students with Disabilities in Their Educational Programs. Occupational Therapy Journal of Research, 21, 115-131. Recuperado el 10 de noviembre de 2013 de: https://lup.lub.lu.se/search/publication/1750448 
Gómez, V. e Infante, M. (2004). Actitudes de los estudiantes de educación hacia la integración de personas con discapacidad y hacia la educación multicultural. CyE: Cultura y Educación, 4 (16), 371-383. Recuperado 5 de septiembre de 2013 de: http://www.ingentaconnect.com/content/fias/cye

Gughwan, C.y Chow, L. (2001). Korean students' differential attitudes toward people with disabilities: an acculturation perspective. International Journal of Rehabilitation Research, 24, 79-81.

Jarvis, C. y French, R. (1990). Attitudes of physical educators toward the integration of handicapped students. Perceptual and motor skllis, 70, 899-902. Recuperado el 10 de octubre de 2013 de: http://www.amsciepub. com/doi/abs/10.2466/pms.1990.70.3.899

Lissi, M., Zuzulich, M., Salinas, M., Achiardi, C., Hojas, A. y Pedrals, N. (2009). Inclusión y discapacidad en contextos universitarios: la experiencia del PIANE-UC. Revista Calidad en la Educación (CSE) (30), 306-324. Recuperado 5 de noviembre de 2013 de: http://www.cpce.cl/ponencias-pucon2010/cat_view/10-programa-anillosoc-01/47-congreso-chileno-de-investigacion-en-ed-superior?start=15

Lissi, M., Zuzulich, M., Salinas, M., Hojas, A. y Medrano, D. (2009). Creencias y actitudes de docentesy estudiantes sin discapacidad respecto a la inclusión de estudiantes con discapacidad en la educación superior. Pontificia Universidad Católica de Chile. Recuperado 18 de octubre de 2013 de: http://www.cpce.cl/ponencias-pucon2010/ cat_view/10-programa-anillo-soc-01/47-congreso-chileno-de-investigacion-en-ed-superior?start=15

López, M. (2006). Actitudes, formación y cambio. En Gómez, A., Gaviria, A. E. y Fernández, I. (coords.). Psicología Social (pp.185-228). Madrid: Sanz \& Torres.

Lucchini, G. (2003). Niños con necesidades educativas especiales. Cómo enfrentar el trabajo en el aula. Santiago: Ediciones Universidad Católica de Chile.

Mella, S. y González, L. (2007). Actitudes de los estudiantes de la salud de la universidad de Chile hacia las personas con discapacidad. Revista Chilena de Terapia Ocupacional, 7 (1), 1-12. Recuperado el 13 de septiembre de 2013 de: http://www.revistaterapiaocupacional.uchile.cl/index.php/RTO/article/viewFile/42/51

Morales, J. et al. (1999). Psicología social. España: McGraw Hill.

Moreno, F., Rodríguez, I., Saldaña, D.y Aguilera, A. (2006). Actitudes ante la discapacidad en el alumnado universitario matriculado en materias afines. Revista Iberoamericana de Educación, 40, 1-12. Recuperado el 10 de noviembre de 2013 de: http://www.rieoei.org/investigacion/1491Moreno.pdf

Novo-Corti, I., Muñoz-Cantero, J. y Calvo-Porral, C. (2011). Análisis de las actitudes de los jóvenes universitarios hacia la discapacidad: un enfoque desde la teoría de la acción razonada. Relieve, 17 (2), art. 5. Recuperado el 4 de septiembre de 2013 de:http://www.uv.es/relieve/v17n2/RELIEVEv17n2_5.htm

Polo, M., Fernández, C. y Díaz, C. (2011). Estudios de las actitudes de estudiantes de Ciencias Sociales y Psicología: relevancia de la información y contacto con personas con discapacidad. Universitas Psychologica, 10 (1), 113123. Recuperado el 12 de septiembre de 2013 de: http://revistas.javeriana.edu.co/index.php/revPsy cho/ article/viewFile/523/785 
Polo, M. y López-Justicia, M. (2006). Actitudes hacia las personas con discapacidad de estudiantes de la Universidad de Granada. Revista Española de Orientación y Psicopedagogía, 17 (2), 195-211. Recuperado el 20 de octubre de 2013 de: http://dialnet.unirioja.es/servlet/articulo?codigo=2199803

Quintero, A., Salazar, R., Padrón, M. y Salazar, L. (2005). Actitud del odontólogo hacia los niños y adolescentes discapacitados. Acta odontológica venezolana, 43, 240-251. Recuperado el 6 de julio de 2013 de: http://www. actaodontologica.com/ediciones/2005/3/actitud_odontologos.asp

Rao, S. (2004). Faculty attitudes and students with disabilities in higher education: a literature revew. College Student Journal, 38, 191-198. Recuperado el 15 de agosto de 2013 de: http://connection.ebscohost.com/c/ articles/14098753/faculty-attitudes-students-disabilities-higher-education-literature-review

Suriá, R. (2011). Comparative analysis of student's attitudes toward their classmates with disabilities. Electronic Journal of Research in Educational Psychology, 9, 197-216. Recuperado el 20 de agosto de 2013 de: http:// rua.ua.es/dspace/bitstream/10045/25924/1/2011_Suria_EJREP.pdf

Upton, T. y Harper, D. (2002). Multidimensional disability attitudes and equitable evaluation of educational accommodations by college students without disabilities. Journal of Postsecondar y Education and Disability, 15, 115-130. Recuperado el 10 de junio de 2013 de: http://webcache.googleusercontent. com/search?q=cache:CKgbWuW2RbwJ:www.ahead.org/uploads/docs/jped/journals/JPEDVol15No2. $\mathrm{doc}+\& \mathrm{~cd}=1 \& \mathrm{hl}=\mathrm{es}-419 \& \mathrm{ct}=\mathrm{clnk} \& \mathrm{gl}=\mathrm{cl}$

Verdugo, M., Arias, B. y Jenaro, C. (1995). Actitudes sociales y profesionales hacia las personas con discapacidad. Estrategias de evaluación e intervención. En Apuntes del Magister en Integración de Personas con Discapacidad. Salamanca: Universidad de Salamanca y Universidad de Valladolid.

Verdugo, M., Arias, B. y Jenaro, C. (1994). Actitudes hacia las personas con minusvalía. Madrid: Inserso. 